\title{
Exploration of the Sport Injury Characteristics and Reasons of Tennis Amateurs
}

\author{
LIU Yuanhai ${ }^{1}$, SHI Guoying ${ }^{2}$, YANG Gang ${ }^{3}$, WANG Zhihui ${ }^{4}$ \\ ${ }^{1,} 3$ Physical Education Institute of Hubei University of Science and Technology, Xianning Hubei, 437100; \\ ${ }^{2}{ }^{4}$ Basic Medical College of Hubei University of Science and Technology, Xianning Hubei, 437100.
}

\begin{abstract}
:
By using the method of questionnaire, literature, data statistics and other research methods, a questionnaire survey was carried out among tennis amateurs in Wuhan, Xiaogan, Xianning. On the basis of knowing the current situation of tennis amateur sports injury on the knowledge, we summarize a conclusion that the tennis injury has seasonal characteristics, the injury site and obvious age characteristic, and analyzes the reasons for sports injury. It has the important guiding significance and reference value to improve the cognition of physical injury for the tennis participants, pay more attention to prevention of tennis injury and raise awareness of prevention.
\end{abstract}

Key words:

tennis; amateur; physical injury; characteristic

Life lies in movement. With the high-speed development of social material civilization, people pay more and more attention to their own health. More and more people participate in the sports, so that the population of participants grows in the global scope with each passing day. Tennis, as the world's second major sports, set entertainment, fitness, competition as a whole, is suitable for different levels of the crowd, and favored by the vast number of sports enthusiasts favor ${ }^{[1]}$. Because the promotion of the sports injury caused by tennis and prevention is not so adequate that participants pay not too much attention on the prevention. Deficient warming-up before exercise, unreasonable batting action and improper footstep movement can cause harm to participants ${ }^{[2]}$. Therefore, we can not ignore the security problem when playing tennis ${ }^{[3]}$. This paper aims to analyze the characteristics and causes of sport injury prevention of sports injury, and provide the theory reference for tennis players.

\section{Research Objects and Methods}

\subsection{Research Objects}

Randomly select samples of 150 tennis amateurs who practice at least once a week and have at least 2 years experience as the object of study from Wuhan, Xiaogan, Xianning. The junior group (8-17 years old) 30 people, youth group (18-44 years old) 60 people, middle-aged group (45-59 years old) 40 people, elderly group (aged over 60) 20 people.

\subsection{Research Methods}

This study mainly adopted questionnaire survey method, literature method, data statistics and logical analysis and other research methods.

\section{Results and Analysis}

2.1 Analysis of the current situation of tennis amateur sports injury

To understand current situation of tennis amateur sports injury, a questionnaire survey that randomly select samples of 150 tennis amateurs was conducted in Wuhan, Xiaogan, Xianning, using face-to-face interviews and immediate recovery, recovery rate and effective rate was $100 \%$, the statistical results are as follows: 
Table 1: Statistics of tennis amateur sports injury situation $(n=150)$

\begin{tabular}{lccccc}
\hline Group Total & Rate of injured $(\%)$ & Rate of never injured(\%) & \multicolumn{2}{c}{ Number of injured Average } \\
\hline Total & 150 & 19.3 & 80.7 & 38 & 0.25 \\
Junior group & 30 & 26.7 & 73.3 & 10 & 0.30 \\
Youth group & 60 & 21.7 & 78.3 & 17 & 0.28 \\
Middle-aged group & 40 & 12.5 & 87.5 & 7 & 0.18 \\
Elderly group & 20 & 15.0 & 85.0 & 4 & 0.20 \\
\hline
\end{tabular}

From Table 1 we can see that the sports injury of the surveyed 150 tennis amateurs was 0.25 on average (palm blisters or hard cocoon not included), while the same repeated injuries happened. Thus, the tennis movement is an antagonism project separated by net, and the confrontation between the two sides have no direct physical contact, but the injury is common. In addition, we can also see from the statistics that the rate of never injured remained at about $80 \%$, which conform to the sports characteristics of net separated competition, namely the confrontation between the two sides have no direct physical contact, resulting in sports injury severity of the probability is very small. Surprisingly, the sports injury incidence rate highest crowd is not in the elderly group, but the youth group. Reflect the intense degree of youth groups involved in tennis is much higher in the elderly group from the side, or can be understood as the old group pay more attention to the protection of their own according to their own conditions.

\subsection{Analysis of the characteristics of tennis amateur sports injury}

To further analyze the damage of tennis amateur sports, make the arrangement and analysis of the cause of sports injury groups, time, main parts and reasons, find tennis amateur sports injury showed obvious seasonal characteristics, site of injury characteristics, age characteristics and various injury factors, concrete analysis is as follows:

2.2.1 Seasonal characteristics of sports injury

Table 2: Statistics of injuries in different seasons $(n=29)$

\begin{tabular}{lcccc}
\hline Season & Spring & Summer & Autumn & Winter \\
\hline Number of injured & 11 & 4 & 6 & 8 \\
Hours of practice per capita & 61.3 & 52.1 & 54.6 & 37.9 \\
Men times of injured & 17 & 5 & 7 & 9 \\
Times of injured per capita & 0.11 & 0.03 & 0.05 & 0.06 \\
\hline
\end{tabular}

A further investigation of 29 interviewers who ever suffered sports injury (statistical results in Table 2), shows that spring (according to the lunar calendar) injured at most times, the per capita wounded 0.11 times, followed by winter, injured 0.06 times per capita. From Table 2 we can also see that the time spent on tennis presents obvious differences in different seasons, the practice time spent in spring, summer, autumn was more than 50 hours, most in spring, 61.3 hours per capita, and the lowest in winter, only 37.9 hours per capita. Through further interviews, analysis the reasons are as follows:

First of all, the number of participants is decided by its characteristics of tennis. The tennis movement mainly belongs to the outdoor sports, although more and more indoor tennis halls appear with the advance of economy, it is minority after all. In contrast, people like to participate in outdoor sports more, bathe in the sunshine and contact with nature, so it is directly affected by weather conditions. Spring, the season of rebirth, appropriate temperature, is a good time for people to stretch the physique through the 
long winter; sunshine time is long in summer, but the temperature is high, it will shatter some people who don't have a strong will; it turns cool and the climate is pleasant in autumn, calling participants back to the tennis court. But the weather is dry and temperature is low, rain and snow sherbet come, hibernating people began a new round of "dwelling life".

Then, the number of injured is affected by the temperature more. In general, blood circulation is quick and muscles, joints, ligaments and other physiological function are in good condition when temperature is high. Despite the large amount of exercise, it won't appear too much injury. On the contrary, low temperature can reactively cause vasoconstriction, muscle stretching reduce obviously, the capacity that nervous system controls over locomotive organs declines. Rough activities in condition of physiological inert can increase the probability of sports injury.

\subsubsection{The main part and age characteristics of} sports injury

The sports injury caused by tennis is decided by the project's unique force mode to some extent. The exerting force order is foot, knee, hip, waist, shoulder, elbow, wrist, fingers in turn, the weak link of which is easy to cause damage in the joint chain. There exists anatomical and physiological weakness [4]. Analyzing the main parts of the 29 people a total of 38 injuries, statistical results are in table 3.

Table 3: Statistical table of the main injury parts $(n=29)$

\begin{tabular}{|c|c|c|c|c|c|c|c|}
\hline \multirow{2}{*}{$\begin{array}{l}\text { Main parts } \\
\text { Group }\end{array}$} & \multicolumn{2}{|c|}{ Upper limbs } & \multicolumn{3}{|c|}{ Torso } & \multicolumn{2}{|c|}{ Lower limbs } \\
\hline & finger & wrist & elbow & shoulder & waist & knee & ankle \\
\hline Junior group & 2 & 5 & 2 & & & & 1 \\
\hline Youth group & 1 & 5 & 3 & 2 & 1 & 2 & 3 \\
\hline Middle-aged group & & 1 & 3 & & 2 & & 1 \\
\hline Elderly group & & 1 & & & 1 & 2 & \\
\hline Total & 3 & 12 & 8 & 2 & 4 & 4 & 5 \\
\hline
\end{tabular}

Joint is the multiple sites in the tennis injury, joint strain is the highest incidence among all kinds of injuries, especially in wrist, elbow joint ${ }^{[5]}$. From Table 3, we can see that the top three main injury parts caused by tennis are wrist, elbow and ankle, which is consistent with the characteristics of batting sports and force mode of the project. In addition, from the age point of view, the main sites of injury are different (see chart 1). The main injury parts in junior group are wrist, elbow and finger in turn, reflecting the week upper limbs strength of juniors, which can easily cause joint strain of upper limbs. The main injury parts of middle-aged group are wrist, elbow and ankle, reflecting that the high-intensity competition, the long crouch and uninterrupted running back and forth increase the load of lower limbs, which can easily cause the injury of ankle. And the easiest injury parts are waist, knee and elbow, reflecting that the physical function of the old declines, which can cause injury of waist, knee and other support zones. 


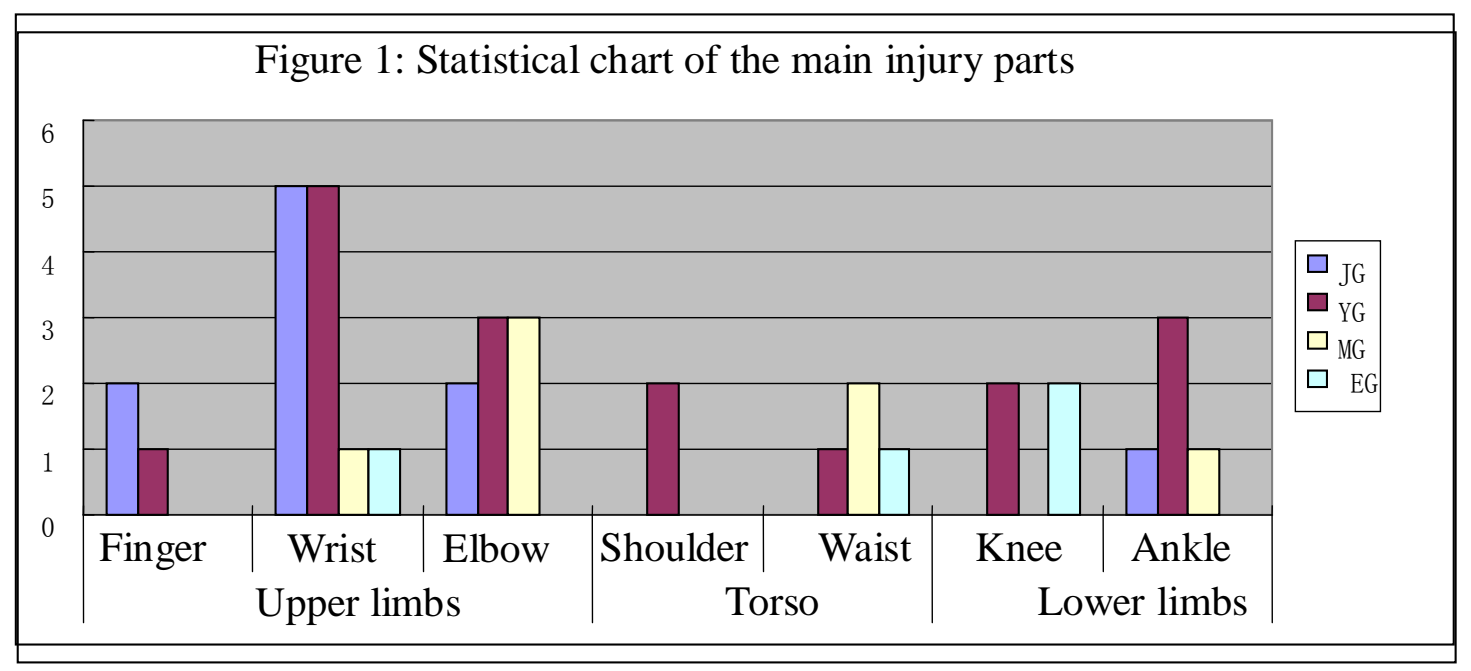

\subsection{Analysis of the causes of tennis amateur sports} injury

Of the 150 respondents 29 people 38 times had different levels of sports injuries. Although the damage probability is very small, only 0.25 per person, the injury still exists after all, and can not be ignored. In order to do well in sports injury prevention work, exploring its reason is an urgent problem to solve. So, a further investigation and interview on the causes of sports injury was carried out, statistical results in Table 4.

Table 4: Statistical table of the investigation on the causes of sports injury $(n=29)$

\begin{tabular}{cccc}
\hline Investigation contents & \multicolumn{3}{c}{ Options } \\
\hline Information of prevention & unaware & a little & very much \\
Percentage $(\%)$ & 31.0 & 48.3 & 20.7 \\
Situation of warm-up & never & sometimes & adequate \\
Percentage $(\%)$ & 27.6 & 48.3 & 24.1 \\
Style of play that causes injury & net rusher & base line & combination \\
Percentage $(\%)$ & 13.8 & 41.4 & 44.8 \\
Segment of sports that causes injury & drive & serve & others \\
Percentage $(\%)$ & 47.4 & 36.8 & 15.8 \\
Type of injury & abrasion or contusion & strain & others \\
Percentage $(\%)$ & 13.2 & 76.3 & 10.5 \\
\hline
\end{tabular}

It is an important cause to sports injury that lack of enough prevention knowledge and warm-up. The investigation of 29 persons who ever suffered sports injury shows players who lack necessary professional prevention knowledge account for $79.3 \%$, and who never warm up account for $75.9 \%$. So, it is necessary to promote the prevention knowledge of tennis. In views of the playing style, the base line style causes the lowest injury, accounting for $41.4 \%$. And the combination style causes the most, clarifying that combination style calls for better physical conditions, no only running wide, but also faster speed and more energetic. In addition, in views of segment of sports and type of injury, drive and serve can easily cause injury. Drive accounts for 47.4. It is easy to cause the wrist and elbow sprain, the finger injury while driving vigorously, immediate running and stopping back and forth can cause sprain of ankle and leg injuries. Serve accounts for $36.8 \%$, heavy serve can cause waist and shoulder strains. And the large 
muscle groups recover slowly after injury, it takes at least three months to recover completely in spite of slightly serious injury, therefore, waist and shoulder should pay special attention to protection.

\section{Conclusion and advice}

\subsection{Conclusion}

(1) The tennis movement is an antagonism project separated by net, and the confrontation between the two sides have no direct physical contact and cannot cause severe injuries, but the injury is common. The sports injury of tennis amateurs has seasonal characteristics, the injury site and obvious age characteristics.

(2) Spring is the season which is easiest to cause injury; the main injury parts are wrist, elbow and ankle in turn, different age group is slightly different; the reasons causing injury are mainly lack of warm-up and awareness of prevention. Vigorous drive is easy to cause the wrist and elbow sprain, heavy serve can cause waist and shoulder strains, and immediate running and stopping back and forth can cause sprain of ankle and leg injuries.

\subsection{Advice}

(1) Tennis has certain requirements for participants, such as power, speed, and physical fitness and so on. Therefore, amateurs should not only learn tennis technology, also should strengthen the physical exercise, the two are interdependent, indispensable.

(2) Arrange the training and competition scientifically, according to their own physical fitness, strength and physical conditions and other circumstance to the reasonable arrangement of the training content and intensity of exercise, prevent playing tennis violently and participating in tennis on physical overdraft situation.

(3) Improve the awareness of prevention and grasp emergency treatment measures, strengthen the treatment after injury and prevent injury again. Make sure appropriate sports environment and don't play tennis on wet playgrounds to prevent slipping. Don't play tennis in arenas where the light is dark to prevent being hit by balls. Equip safe and appropriate equipments when playing tennis.

\section{References:}

[1] SONG Zhiliang. The common injury, treatment and prevention of tennis in our university [J]. Journal of Qiqihar University (PHILOSOPHY AND SOCIAL SCIENCES EDITION), 2006 (9): 174-175

[2] BAO Qin. Common sports injury and its prevention in tennis [J]. Journal of Nanjing Sport Institute (NATURAL SCIENCE EDITION), 2005 (4): 62-65.

[3] ZHANG Yang. Research on common injury and its prevention strategy in universities [J]. Shanxi sports science and technology, 2012 (6): 60-63.

[4] HUANG Hong, WEN Zhijun. Investigation on the characteristics of tennis sports injury and the ability to cope with [J]. Journal of Zhanjiang Normal University, 2008 (12): 115-118.

[5][18] LUAN Lixia, XU Xiangfeng. Investigation of College Students' physical injury of tennis [J]. Journal of Wuhan Sports Institute, 2005, (06):92 95. 